\title{
COUNTABLE GROUPS ARE MAPPING CLASS GROUPS OF HYPERBOLIC 3-MANIFOLDS
}

\author{
Roberto Frigerio and Bruno Martelli
}

\begin{abstract}
We prove that for every countable group $G$ there exists a hyperbolic 3manifold $M$ such that the isometry group of $M$, the mapping class group of $M$, and the outer automorphism group of $\pi_{1}(M)$ are isomorphic to $G$.
\end{abstract}

\section{Introduction}

A hyperbolic manifold here is a connected orientable paracompact manifold (without boundary) equipped with a complete metric of constant sectional curvature equal to -1 . If $M$ is a hyperbolic 3-manifold, $\operatorname{Isom}(M)$ is the group of isometries of $M$, and $\mathcal{M C G}(M)$ the mapping class group of $M$, i.e. the group of isotopy classes of selfhomeomorphisms of $M$. If $G$ is a group, $\operatorname{Out}(G)$ is the outer automorphism group of $G$. We prove here the following:

Theorem 1. For every countable group $G$ there is a hyperbolic 3-manifold $M$ such that:

$$
G \cong \operatorname{Isom}(M) \cong \mathcal{M C G}(M) \cong \operatorname{Out}\left(\pi_{1}(M)\right) .
$$

Together with the fact that there exist uncountably many pairwise non-isomorphic countable groups, Theorem 1 implies the following:

Corollary 2. There are uncountably many non-isomorphic groups that are fundamental groups of hyperbolic 3-manifolds.

Every hyperbolic 3-manifold is the quotient of $\mathbb{H}^{3}$ via the action of a Kleinian group, that is a discrete torsion-free subgroup of $\mathbb{P S L}_{2}(\mathbb{C})$. Theorem 1 implies the following:

Corollary 3. Every countable group is the outer automorphism group of a Kleinian group.

Related results. Theorem 1 is already known for finite groups: Kojima proved in [18] that every finite group is the isometry group of a compact hyperbolic 3-manifold (see also [9]). Moreover, if $M$ is compact hyperbolic, Mostow's rigidity Theorem [27] and Gabai-Meyerhoff-Thurston's results [15] imply that $\operatorname{Out}\left(\pi_{1}(M)\right)$, $\operatorname{Isom}(M)$, and $\mathcal{M C G}(M)$ are isomorphic finite groups. Kojima's Theorem for finite groups has been extended recently to higher dimensions [5], while Winkelmann [33] and Allcock [4]

Received by the editors September 15, 2005.

2000 Mathematics Subject Classification. 57M50 (primary), 20F29, 30F40 (secondary).

Key words and phrases. Kleinian groups, hyperbolic 3-manifolds, special polyhedra.

Both authors are partially supported by the INTAS project "CalcoMet-GT" 03-51-3663. 


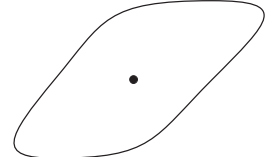

(1)

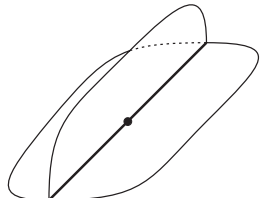

(2)

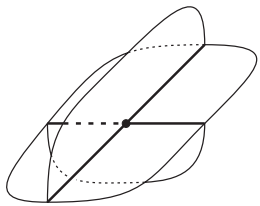

(3)

Figure 1: Neighbourhoods of points in a special polyhedron.

proved that every countable group is the isometry group of a complete hyperbolic surface.

There are many analogues of Corollary 2 concerning topological manifolds of various dimensions. In dimension 2 , there are uncountably many (pairwise non-homeomorphic) paracompact surfaces [14]. On the other hand every paracompact surface supports a complex structure, so Riemann's uniformization Theorem and classical results on Fuchsian groups [21, 29] imply that there exist only countably many isomorphism classes of fundamental groups of surfaces. In dimensions higher than 2, there are uncountably many contractible manifolds $[24,16,11]$, and also uncountably many fundamental groups (see e.g. [16, 28]).

Regarding Corollary 3, Matumoto proved in [23] that every group is the outer automorphism group of some group, while Bumagin and Wise [8] recently showed that every countable group is the outer automorphism group of a two generators group.

Sketch of the proof. We consider some decorated 2-dimensional polyhedra with infinitely many vertices, edges, and faces. We associate to each such polyhedron a hyperbolic 3-manifold by assembling some blocks with geodesic boundary, corresponding to vertices and edges of the polyhedron. We show that the isometry group of the manifold is isomorphic to the combinatorial automorphism group of the polyhedron. It is easy to see that every countable group is the combinatorial automorphism group of one such polyhedron.

If $M$ is a hyperbolic manifold arising from our construction, building on a rigidity result of Keen, Maskit, and Series [17] we show that every automorphism of $\pi_{1}(M)$ is induced by an isometry. This also implies that a self-homeomorphism of $M$ is homotopic to an isometry: it remains to show that it is indeed isotopic to it. This follows from a result of Brown [7], since our $M$ is non-compact and end-irreducible.

The paper is organized as follows. We describe the decorated polyhedra and their associated hyperbolic manifolds in Section 1. A more detailed outline of the proof of Theorem 1 is then given in Section 2. Polyhedra with assigned automorphism group are constructed in Section 3. The needed rigidity results are then proved in Section 4.

\section{From decorated polyhedra to hyperbolic manifolds}

1.1. Decorated polyhedra. A 2-dimensional polyhedron $P$ is special if every point of $P$ has one of the regular neighbourhoods shown in Fig. 1, and if the stratification given by the three types of points gives a cellularization of $P$. That is, points of type 


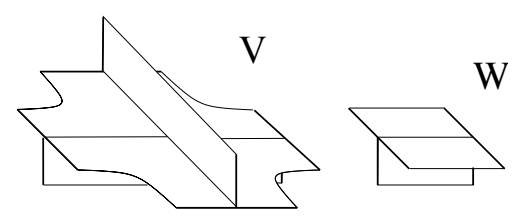

Figure 2: The pieces $V$ and $W$.

(1) form discs - the faces - and points of type (2) form segments - the edges. A vertex is then a point of type (3). In contrast with the usual definition, compactness is not required here: thus $P$ can have infinitely many vertices, edges, and discs (but each has compact closure and is incident to finitely many other vertices, edges, and discs).

A decorated polyhedron is a pair $(P, c)$, where $P$ is a special polyhedron and $c$ : \{edges of $P\} \rightarrow \mathbb{N}$ is a function, which will be called the decoration of $P$. The aim of this section is to describe a recipe for associating to every decorated polyhedron $(P, c)$ an orientable hyperbolic 3 -manifold $M(P, c)$. This will be done by suitably modifying the shadow construction first introduced by Turaev and subsequently explored in $[10$, $9]$.

1.2. From polyhedra to hyperbolic manifolds. Let $(P, c)$ be a decorated polyhedron. Let $P_{0}$ be a regular neighborhood of its 1-skeleton. We subdivide $P_{0}$ into copies of the pieces shown in Fig. 2: we take one piece of type $V$ for each vertex and $c(e)$ pieces of type $W$ for each edge $e$. The "boundary" of $V$ (resp. $W$ ) consists of 4 (resp. 2) $Y$-shaped graphs and 6 (resp. 3) arcs. The following result will be proved in Lemmas 6 and 8 below.

Proposition 4. There are two complete finite-volume hyperbolic 3-manifolds $K$ and $E$ with geodesic boundary such that:

- their boundary consists of thrice-punctured spheres;

- boundary components of $K$ (resp. of E) correspond to $Y$-shaped graphs in $\partial V$ (resp. in $\partial W$ ), with punctures corresponding to endpoints of the graphs;

- annular cusps of $K$ (resp. of $E$ ) correspond to arcs in $\partial V$ (resp. in $\partial W$ );

- the intersections between the boundary components and the annular cusps of $K$ (resp. of $E$ ) correspond to the intersections between the corresponding $Y$ shaped graphs and arcs in $\partial V$ (resp. in $\partial W)$;

- the actions of the isometries on boundary components and cusps induce the isomorphisms:

$$
\begin{gathered}
\operatorname{Isom}^{+}(K) \cong \operatorname{Aut}(\partial V) \cong \mathfrak{S}_{4}, \\
\operatorname{Isom}(E) \cong \operatorname{Isom}^{+}(E) \cong \operatorname{Aut}(\partial W) \cong \mathfrak{S}_{3} \times \mathbb{Z} / 2,
\end{gathered}
$$

- every geodesic thrice-punctured sphere in $K$ (resp. in E) is contained in $\partial K$ (resp. in $\partial E$ ).

We have denoted by $\operatorname{Aut}(Z)$ the combinatorial automorphism group of a graph $Z$, and by $\mathrm{Isom}^{+}$the group of orientation-preserving isometries.

We equip the blocks $K$ and $E$ with arbitrary orientations. We construct from $(P, c)$ an oriented hyperbolic 3-manifold as follows: as we said above, the polyhedron $P_{0}$ 
decomposes into pieces of type $V$ and $W$. Pick blocks of type $K$ and $E$ corresponding to pieces of type $V$ and $W$. For each $Y$-shaped graph we have a thrice-punctured sphere, whose punctures correspond to the endpoints of the graph. Given two geodesic thrice-punctured spheres, every bijection between their punctures is realized by a unique orientation-reversing isometry. The identifications of the $Y$-shaped graphs in $P_{0}$ therefore induce isometries between pairs of boundary punctured spheres, and we use such isometries for gluing all these pairs.

Since any symmetry of $V$ (resp. of $W$ ) translates into an orientation-preserving isometry of $K$ (resp. of $E$ ), there is no ambiguity in the construction, and the result is a complete oriented hyperbolic 3-manifold $M(P, c)$. Annular cusps glue up to toric cusps, which are in natural correspondence with the faces of $P$. Every vertex of $P$ corresponds to a $K$-block. Every edge $e$ of $P$ gives rise to $c(e)$ blocks of type $E$ and $c(e)+1$ thrice-punctured spheres.

Remark 5. If $(P, c)$ is a decorated polyhedron, there are connected complete hyperbolic 3-submanifolds with geodesic boundary $M_{i} \subset M(P, c), i \in \mathbb{N}$ with the following properties:

- $M_{i}$ is the union of a finite number of $K$-blocks and $E$-blocks, for all $i \in \mathbb{N}$;

- $M_{i} \subset \operatorname{int} M_{i+1}$ for all $i \in \mathbb{N}$, and $\bigcup_{i=0}^{\infty} M_{i}=M(P, c)$.

1.3. The block $K$. The rest of this section is devoted to the proof of Proposition 4 . The block $K$ we introduce here has already been used by various authors to construct hyperbolic manifolds. As we mentioned above, Turaev has studied the boundary of the 4-dimensional thickening of a special polyhedron (a shadow), and subsequently Costantino and Thurston have decomposed it into $K$-blocks and solid tori, lying above the vertices and the faces of the polyhedron [10]. The block $K$ has also been used by Minsky to construct the model manifold needed in the proof of the Ending Lamination Conjecture [25], and by Agol to construct non-Haken manifolds of arbitrarily high genus [3].

The piece $V$ is dual to a tetrahedron, which is in turn combinatorially equivalent to an ideal octahedron $O$ with a checkerboard coloring of the faces: see Fig. 3. Note that under this correspondence, the $Y$-shaped graphs and the six $\operatorname{arcs}$ in $\partial V$ are associated respectively to the shadowed faces and the vertices of the octahedron.

Now let us realize $O$ as an ideal regular hyperbolic octahedron, and define $K$ as the geometric object obtained by mirroring $O$ along its white faces. Since the dihedral angles of the regular ideal octahedron are right, $K$ is a complete hyperbolic manifold with non-compact geodesic boundary. As required, $\partial K$ consists of four geodesic thrice-punctured spheres, each of which canonically corresponds to a $Y$-shaped graph in $\partial V$. The six vertices of $O$ give rise to six annular cusps of $K$, which in turn intersect each component of $\partial K$ in (neighbourhoods of) the punctures. Thus a puncture on a component of $\partial K$ canonically corresponds to the intersection point of one $Y$-shaped graph with an arc on $\partial V$ (see Fig. 4).

Lemma 6. We have $\operatorname{Isom}(K) \cong \mathfrak{S}_{4} \times \mathbb{Z} /{ }_{2}$. The isomorphism is obtained by associating to each isometry the induced permutation of the components of $\partial K$ and a number in $\{0,1\}$ saying whether the isometry is orientation-preserving or not. The isometry corresponding to $(i d, 1)$ switches the ideal octahedra in $K$. 


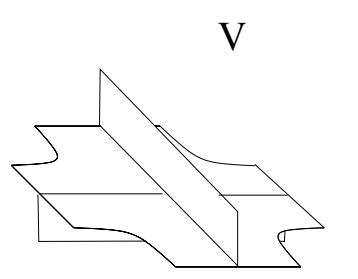

(1)

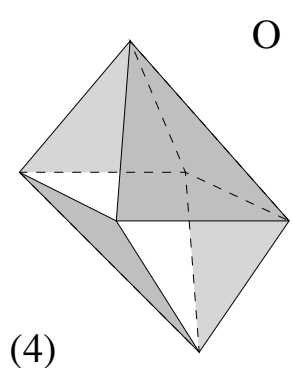

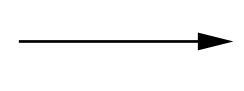
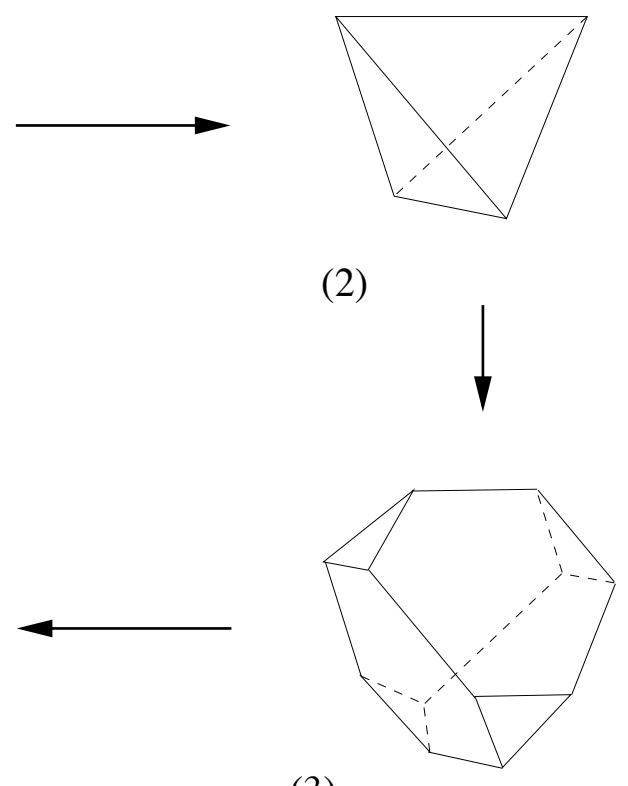

(2)

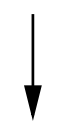

(3)

Figure 3: The polyhedron $P_{0}$ is made of pieces whose boundary consists of $4 Y$ shaped graphs and 6 arcs (1). Each such piece is combinatorially equivalent to a truncated tetrahedron (3), whence to a regular ideal octahedron with checkerboard coloring of the faces (4).

If $S \subset K$ is an embedded geodesic thrice-punctured sphere, then $S$ is a boundary component of $K$.

Proof. The map from $\operatorname{Isom}(K)$ to $\mathfrak{S}_{4} \times \mathbb{Z} / 2$ is surjective, because $K$ inherits the symmetries of the tetrahedron. Concerning injectivity, an isometry in the kernel fixes each thrice-punctured sphere in $\partial K$, each of its punctures, and is orientationpreserving: therefore it fixes $\partial K$ pointwise, and hence the whole of $K$.

Miyamoto proved in [26] that if $N$ is a complete finite-volume hyperbolic manifold with geodesic boundary, then $2 \operatorname{vol}(N) \geqslant-\chi(\partial N) \cdot v_{O}$, where $v_{O}$ is the volume of the regular ideal octahedron. Suppose that $S \subset K \backslash \partial K$ is an embedded thrice-punctured sphere, and let $N$ be the (possibly disconnected) hyperbolic manifold with geodesic boundary obtained by cutting $K$ along $S$. We have $4 v_{O}=2 \operatorname{vol}(K)=2 \operatorname{vol}(N) \geqslant$ $-\chi(\partial N) v_{O}=6 v_{O}$, a contradiction.

1.4. The block $E$. Let $E_{0}$ be the manifold obtained by cutting the double of $K$ along one component of $\partial K$ (by Lemma 6 , the isometry type of this manifold does not depend on the choice of the component of $\partial K$ along which we cut). By construction $E_{0}$ decomposes into two $K$-blocks, has 3 toric cusps, and 3 annular cusps. Moreover, $\partial E_{0}$ is given by two geodesic thrice-punctured spheres. 


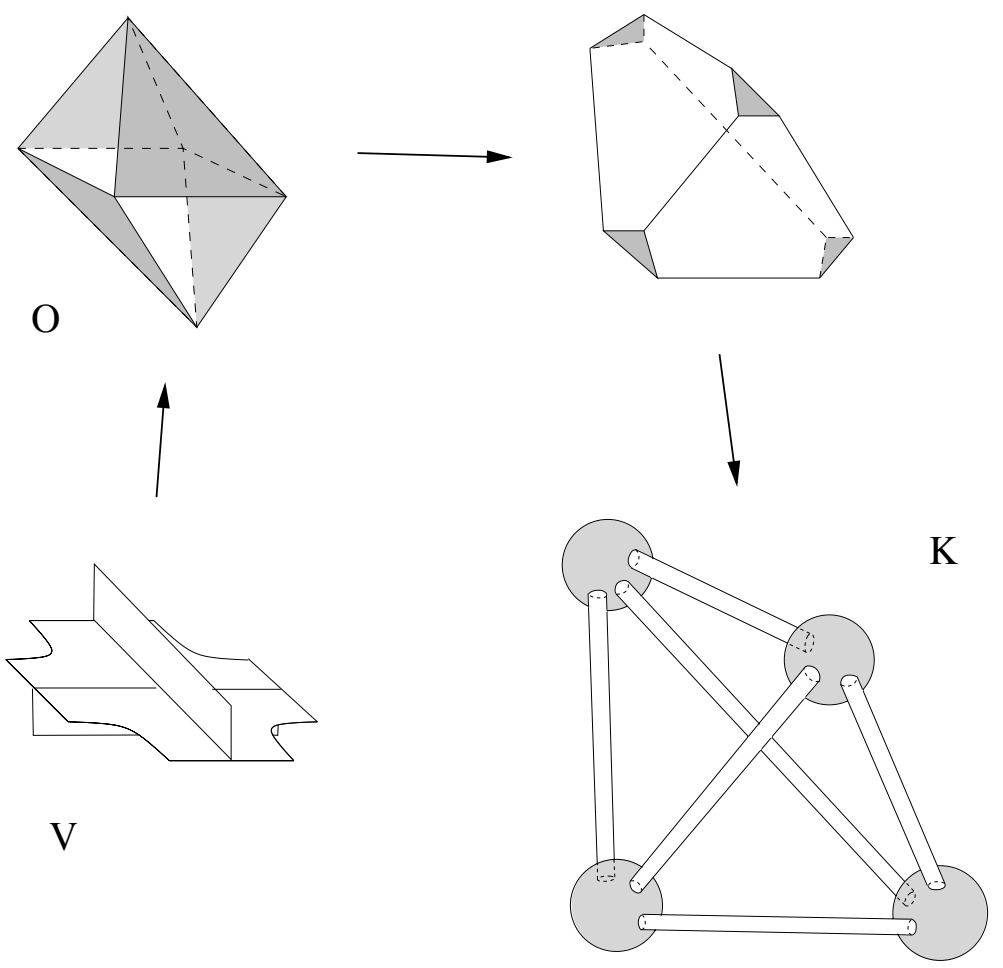

Figure 4: Each piece $V$ gives rise to a block $K$ which is homeomorphic to the complement in $S^{3}$ of four open balls connected by six arcs. The thrice-punctured spheres in $\partial K$ correspond to the $Y$-shaped graphs in $\partial V$.

Lemma 7. We have $\operatorname{Isom}\left(E_{0}\right) \cong \mathfrak{S}_{3} \times \mathbb{Z} / 2 \times \mathbb{Z} / 2$. The isomorphism is obtained by associating to each isometry the induced permutation on the three annular (or toric) cusps, on its two boundary components, and a number in $\{0,1\}$ saying whether it is orientation-preserving or not.

Moreover, if $S$ is a geodesic thrice-punctured sphere embedded in $E_{0} \backslash \partial E_{0}$, then at least one puncture of $S$ lies in a toric cusp of $E_{0}$.

Proof. The map from $\operatorname{Isom}\left(E_{0}\right)$ to $\mathfrak{S}_{3} \times \mathbb{Z} / 2 \times \mathbb{Z} / 2$ is surjective by Lemma 6 , and is injective because an isometry in the kernel fixes $\partial E_{0}$ pointwise.

Suppose $S \subset E_{0} \backslash \partial E_{0}$ is an embedded geodesic thrice-punctured sphere not intersecting the toric cusps of $E_{0}$. Then $S$ should intersect the punctured spheres separating the two blocks in closed geodesics. Since there are no closed geodesics in thrice-punctured spheres, this would imply that $S$ is contained in the interior of one of the two $K$-blocks, against Lemma 6 .

The block $E_{0}$ fullfills all the requirements to be our block $E$, except two: it has an orientation-reversing involution and contains some thrice-punctured spheres (the ones separating the two blocks). We now kill all the redundant isometries and punctured spheres by filling appropriately the three toric cusps. 
We recall that a slope on a torus is an isotopy class of simple closed unoriented curves. Take three disjoint sections of the toric cusps of the same area: their boundaries are Euclidean tori, whose slopes have a definite length. The group $\operatorname{Isom}^{+}\left(E_{0}\right)$ acts on the set of all slopes on the 3 toric cusps. A direct analysis shows that the orbit of any slope $s$ consists of 3 slopes of the same length, each lying on a distinct toric cusp.

An easy doubling argument [13] shows that Thurston's hyperbolic Dehn filling Theorem also applies to hyperbolic manifolds with geodesic boundary. Take then a sufficiently long slope $s$ on a toric cusp, such that the following conditions hold:

(1) the slope $s$ is neither "vertical" or "horizontal", so the corresponding triple is not invariant under orientation-reversing isometries;

(2) on the chosen horospherical sections of the toric cusps, the slopes corresponding to $s$ have length $\ell(s)>12$;

(3) the manifold $E$, obtained via Dehn filling the 3 toric cusps by killing the 3 slopes corresponding to $s$, is hyperbolic with geodesic boundary;

(4) the shortest closed geodesics in $E$ are the cores of the added solid tori.

Lemma 8. Every positive isometry of $E_{0}$ induces an isometry of $E$, and this gives the isomorphism $\operatorname{Isom}(E)=\operatorname{Isom}^{+}(E) \cong \mathfrak{S}_{3} \times \mathbb{Z} / 2$. Moreover, the only geodesic thrice-punctured spheres contained in $E$ are the components of $\partial E$.

Proof. Every positive isometry of $E_{0}$ leaves the triple of killed slopes invariant, and hence extends to a self-homeomorphism of $E$, which is homotopic to an isometry of $E$ by Mostow rigidity for manifolds with boundary [12]. Conversely, every isometry of $E$ fixes the set of the 3 shortest slopes, and hence its complement $E_{0}$. Therefore it is homotopic to an isometry of $E_{0}$ fixing the triple of slopes, which must be orientationpreserving by assumption (1) above.

Suppose we have a geodesic thrice-punctured sphere $S$ in $E$. Since $S$ does not contain closed geodesics, it intersects the three added geodesics in some $k$ points, so $S_{0}=S \cap E_{0}$ is a sphere with $3+k$ punctures inside $E_{0}$. Since $S$ is geodesic, it is easily seen that $S_{0}$ is essential, that is it is incompressible and $\partial$-incompressible, in the sense of [2]. Therefore [2, Theorem 5.1] (or equivalently [20]) implies that $12 k<k \ell(s) \leqslant|6 \chi(S)|=6(1+k)$, whence $k=0$. Thus $S=S_{0}$ lies in $E_{0}$. By [1], $S$ is isotopic to an embedded geodesic thrice-punctured sphere in $E_{0}$ not intersecting the toric cusps of $E_{0}$, whence, by Lemma 7 , to a component of $\partial E_{0}$. This readily implies that $S$ is a component of $\partial E$.

Remark 9. If only the block $K$ were used (as in [9]), then the orientation-reversing involution switching the ideal octahedra of this block would always extend to the whole of $M(P, c)$, thus giving an annoying $\mathbb{Z} / 2$ factor in $\operatorname{Isom}(M(P, c))$. In [9], this factor was killed via Dehn filling, but Thurston's hyperbolic Dehn filling Theorem cannot be applied easily here because the manifold is very big. This is the main reason for introducing the block $E$, which is chiral, i.e. it has no orientation-reversing isometries. The introduction of $E$ also provides some technical semplifications when constructing a polyhedron with the appropriate combinatorial isomorphism group. 


\section{Scheme of the proof of Theorem 1}

We say that a special polyhedron is regular if it has more than two vertices, and every edge in its 1-skeleton has distinct endpoints. A decorated polyhedron $(P, c)$ is big if $P$ is regular and $c(e) \geqslant 4$ for every edge $e$ of $P$. We denote by $\operatorname{Aut}(P, c)$ the group of combinatorial automorphisms of $(P, c)$, i.e. the group of combinatorial automorphisms of $P$ preserving the decoration.

The proof of Theorem 1 consists of two steps:

(1) we show that every countable group is the group of combinatorial automorphisms of some big decorated polyhedron: this is done in Section 3;

(2) for any big decorated polyedron $(P, c)$, we prove in Section 4 bijections of all the following sets:

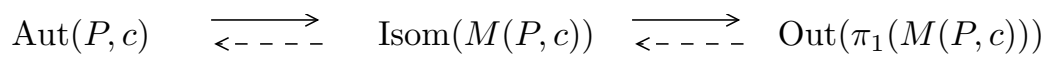

$$
\begin{aligned}
& \begin{array}{lll}
\text { (Prop. 13) } & \downarrow \begin{array}{l}
\hat{1} \\
1
\end{array} \text { (Cor. 17) } & \\
& &
\end{array} \\
& \operatorname{MCG}(M(P, c)) \underset{<---}{\longrightarrow} \mathcal{M C G}^{\text {hom }}(M(P, c)) \\
& \text { (Prop. 21) }
\end{aligned}
$$

We have denoted by $\mathcal{M C G}^{\text {hom }}(M(P, c))$ the group of homotopy classes of selfhomeomorphisms of $M(P, c)$. Maps corresponding to solid arrows are natural, while their inverses, corresponding to dashed arrows, are constructed in Section 4.

\section{Decorated polyhedra with assigned automorphism group}

We prove here that every countable group is the automorphism group of a big decorated polyhedron.

3.1. Special polyhedra with assigned fundamental groups. We begin with the following:

Proposition 10. Every countable group is the fundamental group of a regular special polyhedron.

Proof. A countable group has some presentation with countably many generators and relators. In general, the corresponding polyhedra are constructed by attaching a 1-cell for each generator and a 2-cell for each relator to some simply connected base-space (for instance, a point). Here, since cells need to be locally finite, the base-space needs to be non-compact.

Our base-space is the 2 -skeleton of an infinite wall, i.e. of a strip $\mathbb{R}^{2} \times[0,1] \subset \mathbb{R}^{3}$ tessellated as in Fig. 5. It is a simply connected special polyhedron whose horizontal faces are hexagons and vertical faces are squares. Take an infinite line of consecutive bricks, a point inside the top-face of each brick, and add 1-cells to pairs of consecutive points, as in Fig. 5.

In our presentation, we can suppose that every generator occurs in exactly 3 relators, and once in each $[22]^{1}$. Assign arbitrarily an orientation and a generator to

\footnotetext{
${ }^{1}$ For each generator $g$, substitute its occurrences in the relators with new distinct generators $g_{1}, \ldots, g_{k}$, each occurring once, and add new relators $g_{i} g_{i+1}^{-1}$.
} 


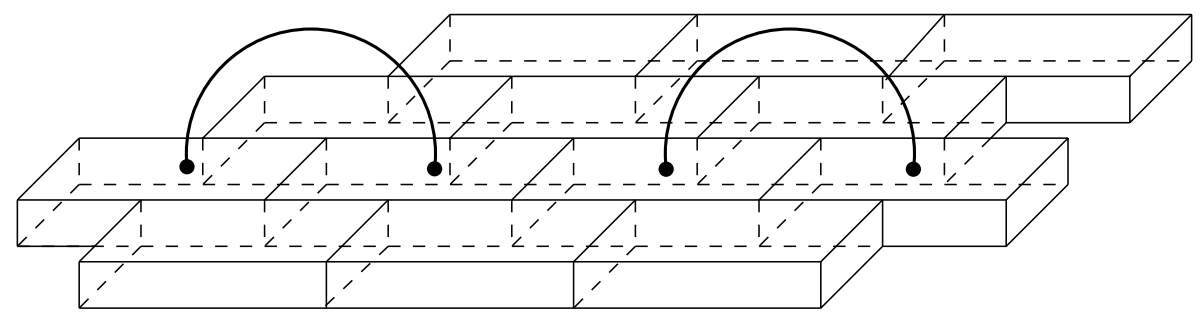

Figure 5: The 2-skeleton of an infinite wall is a special polyhedron, and we add 1-cells to pairs of consecutive bricks in an infinite line.

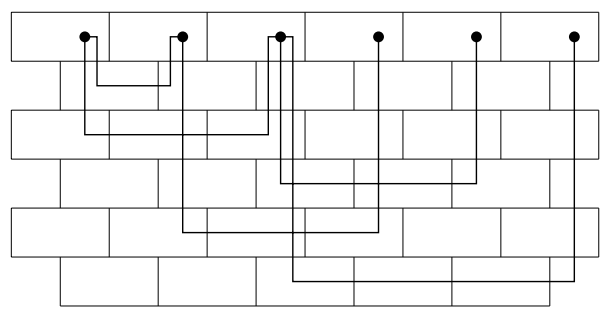

Figure 6: The 2-cells run over the bricks along right-angled paths.

each 1-cell, and attach for each relator a 2-cell running alternatively along a 1-cell (corresponding to a letter) and as a right-angled arc along the wall as in Fig. 6. Each new two-dimensional region is a cell, and three distinct 2-cells run along each 1-cell. Therefore the resulting polyhedron is special. It is easy to see that it is regular.

Proposition 11. Every countable group is the group of combinatorial automorphisms of a big decorated polyhedron.

Proof. In a decorated polyhedron, let us define the colour of a vertex as the 4-uple of colours of the adjacent edges. Let $G$ be a countable group and $P$ be a regular special polyhedron with fundamental group $G$.

Let $e_{0}, e_{1}, \ldots, e_{n}, \ldots$ be an arbitrary ordering of the edges of $P$, and set $c\left(e_{i}\right)=i+4$ for all $i \in \mathbb{N}$. Since $P$ is regular, distinct vertices have distinct colours (because edges have distinct endpoints, and the singular locus of $P$ is not $Q$ ).

Let now $\widetilde{P}$ be the universal cover of $P$, and let $\widetilde{c}$ be the decoration of $\widetilde{P}$ induced by $c$. The fiber over a vertex or edge consists precisely of all vertices or edges of the same colour. Of course the group of deck transformations of $\widetilde{P}$ is isomorphic to $G$. By definition of $\widetilde{c}$, deck transformations are automorphisms. Conversely, every automorphism is a deck transformation, because it must preserve the colours of vertices and edges, and hence the fibers. 


\section{Rigidity results}

4.1. From isometries to combinatorial homeomorphisms of polyhedra. We begin with the following:

Proposition 12. Let $(P, c)$ be a big decorated polyhedron. Then every geodesic thricepunctured sphere in $M(P, c)$ is a boundary component of some block.

Proof. Suppose a geodesic thrice-punctured sphere $S$ is not a boundary component of a block. By Proposition 4, it cannot be contained inside a block, and hence it intersects some boundary component $S^{\prime}$ of some block in a geodesic. The only simple geodesics available in $S$ are lines connecting punctures. Thus $S$ intersects $S^{\prime}$ along a cusp, and it must intersect also every other punctured sphere which is parallel to $S^{\prime}$ along that cusp. Since $(P, c)$ is big, there are at least 4 such punctured spheres: a contradiction, since there are at most 3 pairwise disjoint geodesics on $S$.

Proposition 13. Let $(P, c)$ be a big decorated polyhedron. Then

$$
\operatorname{Isom}(M(P, c))=\operatorname{Isom}^{+}(M(P, c)) \cong \operatorname{Aut}(P, c) .
$$

Proof. Every isometry of $M(P, c)$ preserves the set of all geodesic thrice-punctured spheres, whence, by Proposition 12, the decomposition of $M(P, c)$ into $K$-blocks corresponding to vertices and $E$-blocks corresponding to edges. Together with Proposition 4 , this implies that the group of combinatorial automorphisms of $(P, c)$ is canonically isomorphic to the group of orientation-preserving isometries of $M(P, c)$, so we are left to prove that $\operatorname{Isom}(M(P, c))=\operatorname{Isom}^{+}(M(P, c))$.

Let $\varphi \in \operatorname{Isom}(M(P, c))$ and $E_{1}, E_{2} \subset M(P, c)$ be $E$-blocks with $\varphi\left(E_{1}\right)=E_{2}$. By construction, for $i=1,2$ there exists an orientation-preserving isometry $\psi_{i}$ between $E_{i}$ and the standard block $E$. If $\varphi$ were orientation-reversing, then $\psi_{2} \varphi \psi_{1}^{-1}$ would provide an orientation-reversing element of $\operatorname{Isom}(E)$, against the chirality of $E$.

4.2. Hyperbolic manifolds with geodesic boundary. Let $N$ be a complete finite-volume hyperbolic 3-manifold with non-empty geodesic boundary. Its universal covering $\widetilde{N}$ is isometric to a convex polyhedron of $\mathbb{H}^{3}$ bounded by a countable number of disjoint geodesic hyperplanes [19]. The group of covering automorphisms of $\widetilde{N}$ is a Kleinian group $\Gamma$ acting on $\widetilde{N}$ with $N \cong \widetilde{N} / \Gamma$. Kojima showed in [19] that $N$ is the convex core of $\Gamma$ (see [32] for a definition).

4.3. Maximally parabolic Kleinian groups. Let $G$ be a finitely generated Kleinian group and set $M=\mathbb{H}^{3} / G$. Let $M_{c} \subset M$ be a compact core of $M$ [30] and set $b(G)=-3 \chi\left(M_{c}\right)$. It is shown in [17] that $b(G)$ only depends on the isomorphism type of $G$ as an abstract group, and that $G$ contains at most $b(G)$ conjugacy classes of rank1 maximal parabolic subgroups. A finitely generated Kleinian group $G$ containing $b(G)$ conjugacy classes of rank-1 maximal parabolic subgroups is called maximally parabolic. The following result is a restatement of [17, Theorems I and II], and gives a complete characterization of maximally parabolic Kleinian groups. 
Theorem 14. A finitely generated Kleinian group $G$ is maximally parabolic if and only if its convex core is a complete finite-volume hyperbolic 3-manifold with geodesic boundary, consisting of a finite number of totally geodesic thrice-punctured spheres.

Let $G$ and $G^{\prime}$ be Kleinian groups and suppose $\varphi: G \rightarrow G^{\prime}$ is an isomorphism. We say that $\varphi$ is type-preserving if $\varphi$ sends parabolic elements to parabolic elements and loxodromic elements to loxodromic elements. The following rigidity theorem for maximally parabolic Kleinian groups is taken from [17].

Theorem 15. Let $\varphi: G \rightarrow G^{\prime}$ be a type-preserving isomorphism between two Kleinian groups $G$ and $G^{\prime}$. If $G$ is maximally parabolic, then there exists an element $h \in$ $\operatorname{Isom}\left(\mathbb{H}^{3}\right)$ such that $\varphi(g)=h g h^{-1}$ for all $g \in G$.

4.4. From isomorphisms of fundamental groups to isometries. Let $(P, c)$ be any decorated polyhedron, and let $\Gamma$ be the Klenian group with $M(P, c) \cong \mathbb{H}^{3} / \Gamma$.

Theorem 16. Let $\varphi: \Gamma \rightarrow \Gamma$ be an isomorphism of abstract groups. Then there exists an isometry $g \in \operatorname{Isom}\left(\mathbb{H}^{3}\right)$ with $\varphi(\gamma)=g \gamma g^{-1}$ for all $\gamma \in \Gamma$.

Proof. If $P$ is compact then the conclusion follows from Mostow-Prasad's rigidity Theorem, so we concentrate here on the case when $P$ is non-compact.

The parabolic elements of $\Gamma$ can be characterized as those elements belonging to a $\mathbb{Z} \oplus \mathbb{Z}$ subgroup of $\Gamma$, so $\varphi$ is type-preserving.

Let $M_{i} \subset M(P, c), i \in \mathbb{N}$ be the finite-volume manifolds with geodesic boundary described in Remark 5 , and take a basepoint $x_{0} \in M_{0}$. The map $\pi_{1}\left(M_{i}, x_{0}\right) \rightarrow$ $\pi_{1}\left(M(P, c), x_{0}\right)$ induced by the inclusion is injective, because $M_{i}$ has geodesic (and hence incompressible) boundary. Let $\Gamma_{i}<\Gamma$ be the subgroup corresponding to $\pi_{1}\left(M_{i}, x_{0}\right)$ under the identification $\pi_{1}\left(M(P, c), x_{0}\right) \cong \Gamma$. By Theorem 14, $\Gamma_{i}$ is maximally parabolic, so Theorem 15 implies that for every $i \in \mathbb{N}$ there exists $g_{i} \in \operatorname{Isom}\left(\mathbb{H}^{3}\right)$ such that $\varphi(\gamma)=g_{i} \gamma g_{i}^{-1}$ for all $\gamma \in \Gamma_{i}$. It follows that for all $i \in \mathbb{N}$ the isometry $g_{i} g_{0}^{-1}$ commutes with all the elements in $\Gamma_{0}$. Since $\Gamma_{0}$ is non-elementary, this implies $g_{0}=g_{i}$ for all $i \in \mathbb{N}$, whence $\varphi(\gamma)=g_{0} \gamma g_{0}^{-1}$ for every $\gamma \in \bigcup_{i \in \mathbb{N}} \Gamma_{i}=\Gamma$.

Corollary 17. We have:

$$
\operatorname{Out}\left(\pi_{1}(M(P, c))\right) \cong \operatorname{Isom}(M(P, c)) \cong \mathcal{M C G}^{\text {hom }}(M(P, c)) .
$$

Proof. Since $\Gamma$ is not elementary, the natural map $\pi: \operatorname{Isom}(M(P, c)) \rightarrow \operatorname{Out}\left(\pi_{1}(M, c)\right)$ is injective (in particular, there exists at most one isometry in every homotopy class of self-homeomorphisms of $M(P, c)$ ). By Theorem 16 it follows that $\pi$ is an isomorphism, so we are left to prove that any self-homeomorphism of $M(P, c)$ is homotopic to an isometry.

Let $f$ be a self-homeomorphism of $M(P, c)$. Then there exist an isomorphism $f_{*}: \Gamma \rightarrow \Gamma$ and a $f_{*}$-equivariant lift $\tilde{f}: \mathbb{H}^{3} \rightarrow \mathbb{H}^{3}$ of $f$ (i.e. a lift of $f$ with $\widetilde{f}(\gamma(x))=$ $f_{*}(\gamma)(\widetilde{f}(x))$ for all $\left.\gamma \in \Gamma, x \in \mathbb{H}^{3}\right)$. By Theorem 16, an element $g \in \operatorname{Isom}\left(\mathbb{H}^{3}\right)$ exists such that $f_{*}(\gamma)=g \gamma g^{-1}$ for all $\gamma \in \Gamma$. We now define $\widetilde{F}: \mathbb{H}^{3} \times[0,1] \rightarrow \mathbb{H}^{3}$ by setting $\widetilde{F}(x, t)=(1-t) \cdot \widetilde{f}(x)+t \cdot g(x)$. Being $f_{*}$-equivariant, $\widetilde{F}$ projects onto a homotopy $F: M(P, c) \rightarrow M(P, c)$ between $f$ and an isometry, whence the conclusion. 
Remark 18. In [17], a Kleinian group is by definition a discrete torsion-free subgroup of Isom ${ }^{+}\left(\mathbb{H}^{3}\right)$ with non-empty discontinuity set (see [32] for a definition). However, proofs in [17] also work when dealing with Kleinian groups with full-measure limit set [31]. Moreover, using Remark 5 one could easily prove that if $(P, c)$ is an infinite decorated polyhedron, then every finitely generated subgroup of $\pi_{1}(M(P, c))$ has nonempty discontinuity set, and is actually geometrically finite.

4.5. From homotopy to isotopy. We are now left to prove that homotopic selfhomeomorphisms of manifolds arising from our construction are in fact isotopic. We begin with the following:

Definition 19. Let $M$ be a non-compact manifold. We say that $M$ is end-reducible if there exist a compact set $W \subset M$ and a sequence $\left\{\lambda_{n}\right\}_{n \in \mathbb{N}}$ of simple loops in $M \backslash W$ with the following properties: any compact subset of $M$ intersects only a finite number of $\lambda_{i}$ 's, and each $\lambda_{i}$ is homotopically trivial in $M$ and homotopically non-trivial in $M \backslash W$. A non-compact manifold is end-irreducible if it is not end-reducible.

Remark 20. The notion of end-irreducibility was introduced in [6]. Our definition of end-irreducibility is proved to be equivalent to the original one in [6, Lemma 3.1].

It is shown in [7] that two homotopic self-homeomorphisms of an end-irreducible non-compact manifold are in fact isotopic. Therefore, in order to conclude the proof of Theorem 1 we only need to show the following:

Proposition 21. Let $(P, c)$ be an infinite decorated polyhedron. Then $M(P, c)$ is end-irreducible.

Proof. Suppose $M=M(P, c)$ is end-reducible, and let $W$ and $\left\{\lambda_{n}\right\}_{n \in \mathbb{N}}$ be as in Definition 19. By Remark 5 , there exists a complete finite-volume hyperbolic manifold with geodesic boundary $M_{W} \subset M$ containing $W$. Let $C_{1}, \ldots, C_{j}$ be the toric cusps of $M$ meeting $M_{W}$. Up to rescaling, we can suppose that each $C_{l}$ is disjoint from $W$. Let $T_{l} \subset M$ be the Euclidean torus bounding $C_{l}$, and set

$$
M_{W}^{\prime}=\left(M_{W} \backslash\left(C_{1} \cup \ldots \ldots C_{j}\right)\right) \cup\left(T_{1} \cup \ldots \cup T_{j}\right) .
$$

Since $M_{W}^{\prime}$ is compact, there exists $N \gg 0$ such that $\lambda_{N} \cap M_{W}^{\prime}=\emptyset$. Suppose $\lambda_{N} \subset C_{l}$ for some $l$. Being non-trivial in $M \backslash W$, the loop $\lambda_{N}$ is non-trivial in $C_{l}$, whence in $M$, since the map $i_{*}: \pi_{1}\left(C_{l}\right) \rightarrow \pi_{1}(M)$ induced by the inclusion is injective: a contradiction. We can thus suppose $\lambda_{N} \subset M \backslash M_{W}$. Since $\lambda_{N}$ is non-trivial in $M \backslash W$, it is non-trivial in $M \backslash M_{W}$. Moreover, each surface in $\partial M_{W}$ is geodesic, whence incompressible in $M$, and this readily implies that for any component $L$ of $M \backslash M_{W}$ the map $i_{*}: \pi_{1}(L) \rightarrow \pi_{1}(M)$ induced by the inclusion is injective. This gives $i_{*}\left(\lambda_{N}\right) \neq 1 \in \pi_{1}(M)$ : a contradiction. 


\section{References}

[1] C. Adams, Thrice-punctured spheres in hyperbolic 3-manifolds, Trans. Amer. Math. Soc. 287 (1985) 645-656.

[2] I. Agol, Bounds on exceptional Dehn filling, Geom. Topol. 4 (2000) 431-449.

[3] S Small 3-manifolds of large genus, Geom. Dedicata 102 (2003) 53-64.

[4] D. Allcock, Hyperbolic surfaces with prescribed infinite symmetry groups, Proc. Amer. Math. Soc. 134 (2006) 3057-3059.

[5] M. Belolipetsky and A. Lubotzky, Finite Groups and Hyperbolic Manifolds, Invent. Math. 162 (2005) 459-472.

[6] E. M. Brown and T. W. Tucker, On proper homotopy theory for non-compact 3-manifolds, Trans. Amer. Math. Soc. 188 (1974) 105-126.

[7] M. S. Brown, Constructing isotopies on non-compact 3-manifolds, Trans. Amer. Math. Soc. 180 (1973) 237-263.

[8] I. Bumagin and D. T. Wise, Every group is an outer automorphism group of a finitely generated group, J. Pure Appl. Algebra 200 (2005) 137-147.

[9] F. Costantino, R. Frigerio, B. Martelli, and C. Petronio, Triangulations of 3-manifolds, hyperbolic relative handlebodies, and Dehn filling. Math. GT/0402339, to appear in Comment. Math. Helv.

[10] F. Costantino and D. P. Thurston, 3-manifolds efficiently bound 4-manifolds. Math. GT/0506577.

[11] M. L. Curtis and K. W. Kwun, Infinite sums of manifolds, Topology 3 (1965) 31-42.

[12] R. Frigerio and C. Petronio, Construction and recognition of hyperbolic 3-manifolds with geodesic boundary, Trans. Amer. Math. Soc. 356 (2004) 3243-3282.

[13] M. Fujii and S. Kojima, Flexible boundaries in deformations of hyperbolic 3-manifolds, Osaka J. Math. 34 (1997) 541-551.

[14] M. Fujita and M. Shiota, Topological types of Pfaffian manifolds, Nagoya Math. J. 173 (2004) $1-22$.

[15] D. Gabai, G. R. Meyerhoff, and N. Thurston, Homotopy hyperbolic 3-manifolds are hyperbolic, Ann. of Math. 157 (2003) 335-431.

[16] L. C. Glaser, Uncountably many contractible open 4-manifolds, Topology 6 (1965) 37-42.

[17] L. Keen, B. Maskit, and C. Series, Geometric finiteness and uniqueness for Kleinian groups with circle packing limit sets, J. Reine Angew. Math. 436 (1993) 209-219.

[18] S. Kojima, Isometry transformations of hyperbolic 3-manifolds, Topology Appl. 29 (1988) 297307.

[19] - Polyhedral decomposition of hyperbolic manifolds with boundary, in Proc. Workshop Pure Math., Vol. 10, 37-57 (1990).

[20] M. Lackenby, Word hyperbolic Dehn surgery, Invent. Math. 140 (2000) 243-282.

[21] A. M. Macbeath and A. H. M. Hoare, Groups of hyperbolic crystallography, Math. Proc. Cambridge Philos. Soc. 79 (1976) 235-249.

[22] M. A. Marcus, All countable groups have cubic presentations, Proc. Amer. Math. Soc. 93 (1985) 411-413.

[23] T. Matumoto, Any group is represented by an outerautomorphism group, Hiroshima J. Math. 19 (1989) 209-219.

[24] D. R. McMillan, Some contractible open 3-manifolds, Trans. Amer. Math. Soc. 102 (1962) 373-382.

[25] Y. N. Minsky, Combinatorial and geometrical aspects of hyperbolic 3-manifolds, in C. U. Press, editor, Kleinian groups and hyperbolic 3-manifolds, Vol. 299 of London Math. Soc. Lecture Note Ser., 3-40, Warwick, 2001 (2003).

[26] Y. Miyamoto, Volumes of hyperbolic manifolds with geodesic boundary, Topology 33 (1994) 613-629.

[27] G. D. Mostow, Quasi-conformal mappings in n-space and the rigidity of hyperbolic space forms, Inst. Hautes Études Sci. Publ. Math. 34 (1968) 53-104.

[28] R. Myers, Uncountably many arcs in $S^{3}$ whose complements have non-isomorphic, indecomposable fundamental groups, J. Knot Theory Ramifications 9 (2000) 505-521. 
[29] N. Purzitsky, A cutting and pasting of noncompact polygons with applications to Fuchsian groups, Acta Math. 143 (1979) 233-250.

[30] G. P. Scott, Compact submanifolds of 3-manifolds, J. London Math. Soc. 7 (1973) 246-250.

[31] C. Series. Private communication.

[32] W. P. Thurston, The geometry and topology of 3-manifolds, Princeton (1979). Mimeographed notes.

[33] J. Winkelmann, Realizing countable groups as automorphism groups of Riemann surfaces, Documenta Math. 7 (2002) 413-417.

Dipartimento di Matematica, Università di Pisa, Largo B. Pontecorvo 5, 56127 Pisa, ITALY

E-mail address: frigerio@mail.dm.unipi.it, martelli@dm.unipi.it 